\title{
ALLERGY IN SKIN DISEASE
}

\author{
By C. D. Calnan, M.B., M.R.C.P. \\ Consultant Dermatologist, St. Fohn's Hospital for Diseases of the Skin, London \\ Physician, Skin Department, Royal Free Hospital, London
}

The skin has considerable importance in relation to allergy. Many allergic disorders have manifestations which can be seen in the skin, apart from those in which the skin itself is the shock organ. And because the skin is so easily accessible, it can be used for various allergy test procedures, either clinically or experimentally. Notable advances have been made in the whole field of allergy and immunology in recent years, particularly in relation to the skin; and some of them subsequent to the review six years ago of the basic mechanisms and the role of allergy in clinical medicine in this journal by Pepys (1953). In spite of this, however, much controversy remains and many problems are still unsolved.

\section{Urticaria}

Urticaria is the clinical prototype of the allergic reaction in the skin. Sir Henry Dale's discovery of $H$ substance or histamine was the starting point for numerous investigations; the fact that histamine could produce vasodilatation and increased capillary permeability-in fact, all the elements of Sir 'Thomas Lewis' triple response of erythema, wheal, and local axon flare-tended for a long time to identify this amine as the chemical mediator of the allergic reaction. The demonstration by Riley and West (1953) of the mast cells in the skin and other tissues as the principal store of histamine, came after the dominant role of histamine was being challenged by 5 -hydroxytryptamine (5-HT or serotonin), proteolytic enzymes, and kinins. The use of histamine liberating substances, antihistamine drugs, and other blocking agents have shown the problem to be much more complex than was originally suspected (Inderbitzen, I955).

From the clinician's point of view, urticaria is best divided into acute and chronic forms, the acute cases consisting of one or more relatively brief episodes, and the chronic cases being those which persist with only short intervals of freedom beyond an arbitary period of about three to six months. Many, but not all, of the acute cases can be traced to causative agents, such as a food, inhalant, or other foreign protein allergen. They may be shown to be dependent on the presence of a circulating antibody in the serum by means of skin tests (prick, scratch, or intradermal) with the appropriate antigen, or by the passive transfer technique. The latter method is not now recommended because of the very real risk of serum hepatitis, which may be severe and sometimes fatal. Most of the patients who have circulating antibodies to food antigens give such a precise history of urticarial swelling of the mouth and lips, with or without vomiting, only and on every occasion when they eat that particular food, that skin testing is not normally required for diagnosis. Patients, however, vary enormously in their sensitivity; some may react to raw or lightly cooked eggs but not to eggs used in cakes, pastries, and so on, whilst others react to eggs in any form; some patients may be so sensitive to fish protein that they cannot handle paper or plates which have been in contact with fish, without developing urticarial swelling of their hands (but never an eczematous reaction, even in atopic subjects). Not infrequently there is some disparity between a patient's clinical history and the skin tests with food antigens, since allergic sensitivities can be subclinical. Important, on the other hand, is the fact that hydrolysates of some shellfish, fruits and possibly other foods can liberate histamine. This may be the explanation of some anomalous and irregular skin test results. Experimental work has shown that a wide variety of different substances can release histamine without any antigen-antibody reaction taking place (Paton, 1956). Some examples are snake venoms and animal toxins, proteolytic enzymes like trypsin, tween 20 and bile salts, egg white, dextran, horse serum, and polyvinyl pyrollidone, alkylamines and compound $48 / 80$. In addition numerous drugs have this property to some degree, for example, morphine, pilocarpine, codeine, atropine, quinine, curare, stilbamidine, apresoline, and aspirin (Winkelmann, I957, Pepys, r95I). It is noteworthy that in chronic urticaria, a specific antigen is rarely found to be the cause, and skin testing is 
unrewarding. It is possible that one of the numerous histamine release mechanisms is operating. Penicillin is an important cause of urticaria, and it tends to persist for a period of a few weeks or even months after the drug has been stopped. Skin testing is unhelpful and antibodies can rarely be demonstrated. Patients may be so extremely sensitive to it as to react to the small amounts present in milk, consequent upon the use of the drug for treatment of mastitis in cows (Zimmerman, I959). Considerable benefit has been derived from penicillinase preparations, although there is the risk of such agents themselves being antigenic. In both acute and chronic urticaria drug sensitivity should always be considered as a possible cause. It is said to account for $10 \%$ of cases. Aspirin can be allergenic, but in the writer's experience it appears to act more frequently as a histamine liberator only thereby aggravating many instances of chronic urticaria.

Serum sickness is infrequently seen with the diminished use of specific sera and with the special treatment of foreign sera, but a number of examples were encountered last year following the widespread inoculations with Asian influenza vaccine prepared from virus grown on eggs. No description of the condition can better the classical monograph by von Pirquet and Schick (1905), which was recently translated into English by the latter author. Oedema and urticaria are the important cutaneous components of the syndrome and they may persist for some months. Penicillin may produce it, in addition to the more severe and sometimes fatal anaphylactoid reaction, which is normally only seen with protein antigens; it is more frequently encountered in patients with the atopic diathesis.

For treatment, a wide variety of anti-histamine drugs is available. The most suitable one may have to be found by trial and error for each patient, but it is important to prescribe adequate dosage without incapacitating side effects; to give the drug at the times of day when the patient expects his urticaria to be worse; and to continue the drug for a few weeks after the urticaria has ceased. The itching of urticaria is associated with the erythematous flare rather than the wheal; the chemical mediator of the itch sensation is almost certainly a proteolytic enzyme (Shelley and Arthur, 1955). But the precise inter-relationship of the enzyme and histamine is, as yet, unclarified. Steroids are also effective when given orally or parenterally, but their use should be restricted to the most severe cases, and they are best avoided in chronic urticaria.

\section{Atopic Dermatitis}

The term atopic dermatitis was introduced by
Coca (I93I) to delineate a particular pattern of $\frac{3}{\infty}$ eczema which was especially liable to be seen in $c$. association with a personal or a family history of $\overrightarrow{\vec{F}}$ allergic asthma or hay fever. Its manifestations $\stackrel{\oplus}{\rightarrow}$ can be very varied in infancy, childhood, adolescence and adult life. The term has proved a useful unifying concept, in this regard, from the dermatologist's point of view, although not universally accepted. It has, however, been responsible for a continuous controversy, which remains unresolved. The subject was recently debated by Mayer (1957) and Jadassohn (1957) at the Third International Collegium of Allergology in London. The hypersensitivity basis of allergic contact dermatitis is generally accepted as a cause and effect relationship. The simple chemicals or non-protein substances involved behave as incomplete antigens, although the delayed type ' antibodies' to which they give rise, do not conform to the criteria of the classical type of precipitation or agglutination antibodies, and they have not been satisfactorily separated from cellular elements. Nevertheless, these ' antibodies' appear to be the effective means by which the contact dermatitis reaction is produced. In the case of atopic dermatitis, on the other hando one finds that an appreciable proportion, thoug not all, of the patients have immediate type antio bodies in their serum similar to those found in asthma, hay fever, and urticaria. Their presence can be demonstrated by the usual prick, scratch, intradermal, or passive transfer tests. The crux of the matter is the relationship of these serum antibodies to the dermatitis. The usual dermatological viewpoint is that such antibodies relate to the patient's asthma, hay fever, and urticarial tendencies only, and not to their dermatitis (Schnyder, 1957), while many allergists believe that there is a causal relationship. The issue has been obscured by many observations. Sulzberger (1940) said 'we have never succeeded in producing the dermatosis de novo, and we know of no regularly successful production of the dermatosis by deliberate exposure to the presumptive allergen during dermatosis-free periods.' In dietary studies, Cooke (1944) was unable to detect any exacerbation of eczema from the ingestion of foodstuffs to which the patient gave a positive wheal reaction. Similar views are expressed by Adamson and MacLeod (1926), O'Leary (1953), and Rostenberg (1955). Evidence to the contrary has been cited by Engman, Weiss and Engman (1936), Tuft (1949) and Herrmann (1946). It must be emphasized, however, that the evidence advanced by these latter authors shows that exposure to atopic reagins may produce itching and then secondarily exacerbate or aggravate existing dermatitis; but this is not comparable to the cause 
and effect relationship of allergic contact dermatitis or of hay fever. The various theories concerning the pathogenesis of the atopic state have been well reviewed by Rostenberg (1955). More recently Strauss and Kligman (1957) have produced experimental data to show that there may be a mechanism by which food and inhalant allergens can provoke flares of an existing but aetiologically unrelated dermatitis. They found that the intranasal, subcutaneous, or surface application of such reagins in sensitive patients influenced the behaviour of areas of poison ivy dermatitis produced by known quantities of the specific allergen (pentadecylcatechol). The contact dermatitis was shown to be aggravated, made to persist longer, and to produce lichenification, perhaps as a result of scratching. It would appear, therefore, that the reagins occupy a secondary rather than a primary rôle in atopic dermatitis.

One must agree with Mayer (1957) when he says that the disagreement between allergists and dermatologists is perpetuated primarily by misconceptions and misinterpretations. However, the writer is unable to support his contention that atopic dermatitis and contact dermatitis are quite closely related, and that they are both of allergic origin. It is accepted that simple chemical compounds such as paraphenylenediamine, picryl chloride or quinine, can induce the formation of both immediate and delayed type antibodies, with their appropriate clinical manifestations. Kligman (1958) has recently emphasised the not infrequent occurrence of immediate type reactions in contact sensitization to poison ivy. Chase (1954) has showed that the type of antibody produced depended in part on the route of administration, and Mayer (I957) believes it to be associated with whether the chemical hapten links with a fibrous or globulin protein as a carrier. His view is that if the chemical is applied to the skin it is most likely to combine with keratin or collagen protein and so form delayed type antibodies; while if the chemical is injected intravenously, it would combine with albumen or globulin protein and so form immediate type antibody. There is, however, incomplete proof of this theory. It is indisputable that one chemical substance may induce both types of antibody. The point at issue is that, while the delayed type antibodies are a sine qua non for the development of allergic contact dermatitis, there is ample evidence that atopic dermatitis can occur in the absence of, and independently of any demonstrable immediate type antibodies. Atopic dermatitis, in fact, is not primarily an allergic dermatitis at all. The atopic state or diathesis is an inherited abnormality manifested as a syndrome of associated conditions - a particular clinical pattern of eczema, asthma, hay fever, and urticaria (dependent on reagin antibodies in the plasma); certain physiological stigmata (Kierland, I955) such as white dermographism and abnormal sweat gland responses; and a special type of cataract. Gans (1956) believes it is linked to the leptosome type of human physique.

\section{Contact Dermatitis}

Contact dermatitis is a unique allergic response of the skin. It is more highly developed in man than in any other animal, perhaps as a defence mechanism compensating for the loss of a universal hair covering. From the immunological standpoint it is an example of the delayed type of hypersensitivity, and is not normally associated with any demonstrable antibodies in the plasma. Our understanding of the allergic mechanisms involved in contact dermatitis has been advanced commensurately with the increased biological and immunological interest and research in delayed hypersensitivity reactions over the past decade. Most of the work has been done in animals, especially the guinea-pig, but recent attempts have been made to study the reaction experimentally in man. The skin of guinea-pigs, when sensitized by contact with simple chemical compounds, does not react with the histological response of the dermatitis reaction found in man, except over the skin of the nipple. However, this is due to an anatomical and not an immunological difference. If the skin is thickened (i.e. made acanthotic) by previous treatment with a suitable agent (such as lauryl sulphate) the guinea-pig' sskin can be made to produce analogous histological changes (Jadassohn, I957), but in most experimental work this procedure is unnecessary. Bloch, Mayer, Landsteiner, and many others have shown that the guinea-pig can be readily sensitized by the repeated application to the skin of relatively simple chemicals, such as primula extracts, paraphenylenediamine, dinitrochlorbenzene, picryl chloride and paranitrodimethylaniline. It has been assumed that these chemicals are haptens which must be conjugated with protein to form a complete antigen. Such a conjugation can be produced in vitro and has been shown to occur in vivo. But the in vitro conjugated chemical is not a more effective antigen than the simple chemical alone, and when injected the conjugated chemical does not usually produce contact type sensitivity-it has to be applied to the skin surface. As mentioned previously, Mayer (I957) feels that the chemical must be conjugated with a fibrous rather than a globular protein in the skin in order to produce delayed as opposed to immediate type antibodies.

Much recent interest in this field has centred 
round the role of the lymphocyte and the regional lymph nodes. Landsteiner and Chase (1942) originally showed that contact sensitization in guinea-pigs could be transferred by means of lymphocytes, and this important observation has been amply confirmed whether the lymphocytes are obtained from circulating blood, thymus, lymph nodes, spleen, thoracic duct, or by parabiosis. The experiments of Haxthausen (I939) are particularly interesting. He transplanted skin from sensitized to non-sensitized animals and vice versa, and repeated the operation in identical human twins. The results demonstrated ' recipient dominance' i.e. if a sensitized animal received a graft from a non-sensitized one, that graft showed sensitivity after it had become firmly established; and if a non-sensitized animal received a graft from a sensitive one, the graft ceased to show any reactivity. Hence the skin was demonstrated to be mainly important as a target organ and not to play any part in the immunological process, apart from its still undefined role in initial sensitization. Frey and Wenk (1956) have studied the problem with an ingenious skin explant which is connected to the flank of the guinea-pig only by a neurovascular bundle, and by a slender bridge of skin containing lymphatic channels. They found that the application of a suitable chemical to the main skin of the animal could sensitize the explant only when the direct vascular supply was intact; whereas, when the chemical was applied to the explant, sensitization of the whole animal depended on the integrity of the lymphatic channels and the regional lymph nodes.

There is now little doubt that the lymph nodes are the principal sites of manufacture of delayedtype antibodies, and their cells can transfer sensitivity to non-sensitive animals. Further, lymph node cells can produce antibodies of delayed type hypersensitivity in vitro, as shown by Harris, Harris and Farber (1956) in rabbits using antigenic material from Shigella paradysenteriae. If this can be shown to be true of the contact dermatitis reaction in man, the dominating role of the regional lymph glands will be established. That human contact dermatitis can be transferred by lymphocytes, has been confirmed by Epstein and Kligman (1957). They emphasized that the important requirements are (I) a very high degree of sensitivity in the donor, (2) a large number of lymphocytes (probably about 200 million), (3) a very potent allergen, and (4) a recipient who is genetically susceptible to being sensitized. An intriguing aspect of sensitization by lymphocyte transfer is that some of the recipients remained sensitive for several months-long after all the donated cells must have been destroyed by the homograft reaction. It would seem that some form of active sensitization, as well as passive transfer, had been achieved. The details of the process are not clear, but it is suggested that the intra-lymphocytic antibody protein is incompletely metabolized when such cells die, and is taken up up by young cells and re-utilized by them as functioning antibody (Hamilton, 1958). Because such antibodies behave so differently from classical precipitating or agglutinating antibodies, some immunologists feel they should have a separate designation and refer to them as " transfer factors.' Pappenheimer (1958) has put forward a theoretical scheme for the way in which such transfer factors are released from lymphocytes which contain them and are then able to activate new lymphocytes which take them up. Such a scheme may be useful as a working hypothesis, but it yet awaits the necessary proof.

Kligman's (1958) extensive clinical and experimental study with poison ivy (Rhus) has emphasized important aspects of contact dermatitis. White persons are more readily sensitized than the Negro, Puerto Rican or Hawaiian. Pre-pubertal children are much more easily sensitized with I per cent. pentadecyl-catechol (one of the poison ivy allergens) than are adults. But this difference may be due to previous (unknown) exposure i the case of the latter. Prior exposure has an im portant influence on later sensitization. Patientọ with atopic eczema, asthma, or hay fever, are not more prone to rhus dermatitis. All contact sensitivity has a natural tendency to diminish with age, and this is probably part of a general biological phenomenon, as illustrated by the diminution of tuberculin sensitivity with age. Contact sensitivity may be sub-clinical, as when patients think they are immune after failure to develop dermatitis from a weak exposure to an allergen, although a patch test with a stronger concentration is positive. A significant difference from other types of allergy is that fresh exposure to the antigen does not ordinarily increase the titre of sensitivity, which appears to be determined mainly during the initial sensitization phase. The factors responsible during that period are unknown, but a genetic predisposition is probably one of them. A severe attack of dermatitis not infrequently makes the skin supersensitive in a non-specific fashion (the socalled status eczematicus) and false positive patch tests can be elicited. The reverse condition also occurs, though rarely. The skin becomes refractory and may not react to the specific allergen which has caused the dermatitis, except in very high concentrations. Whether the skin is too exhausted to react or whether there is an immunological change is uncertain.

There have been numerous discussions since Civatte described his "vesiculette primordiale" 
as to whether the dermis or the epidermis is the site of the initial reaction in contact dermatitis. It now seems clear that the process begins around the blood vessels of the dermis and that all contact dermatitis is a combined dermal and epidermal reaction. Kligman has shown that the dermis will still react to contact allergens after the epidermis has been removed. The terms dermal and epidermal sensitivity used by dermatologists to denote immediate and delayed type allergic reactions are anachronisms. Epstein (1956) has raised a further difficulty by coining the term ' dermal contact dermatitis' for cases in which the patch test is negative, but an intradermal test gives a delayed positive result. Many such cases can be explained by lack of penetration of the allergen through the epidermis, when present in low concentration, examples being neomycin (Calnan and Sarkany, 1958), and eosin (Calnan and Sarkany, 1957). Epstein (1958), however, maintains that there is a different immunological mechanism involved.

Desensitization or hyposensitization against contact dermatitis can be achieved but it is rarely indicated in practice, having regard to the time, care, and side effects involved. It may be justified in certain circumstances such as with nurses suffering from streptomycin or penicillin dermatitis (Wilson, 1958), or with North Americans afflicted by rhus allergy. Kligman (1958) has evolved a satisfactory scheme for hyposensitization to poison ivy by means of graduated oral doses of cashew nut shell oil (which contains related allergens), and has demonstrated a lowering of the patient's sensitivity titre. $\mathrm{He}$ rightly warns against accepting clinical improvement as evidence that a patient has been immunologically desensitized. Once hyposensitization has been achieved, it unfortunately wanes, and the original sensitivity slowly returns. This is in contrast with the difficulty of re-establishing a contact sensitivity in a person who has lost it naturally with old age or over the passage of years.

\section{Drug Reactions}

The commonest manifestations of drug sensitivity are in the skin. There are many different causative mechanisms in drug reactions, but allergy is believed to be the basis of the largest group, although proof is frequently lacking. Evidence of a specific serum antibody has been provided in the case of sedormid thrombocytopenic purpura by the work of Ackroyd (1949) and other drugs have been shown capable of linking with platelets to form a complete antigen. A notable dividend of Ackroyd's work is the diagnostic in vitro test for serum antibody. The claim of Hoigné et al. (1956) to be able to identify specific reacting factors in the serum of patients with drug sensitivities, has not yet been satisfactorily confirmed, in spite of attempts by several workers. Hoigné and his colleagues used a nephalometer to detect an increase in turbidity when the serum and a solution of the drug were mixed in optimal concentrations. The presence of antibodies has not been confirmed in any other form of drug sensitivity except thrombocytopenic purpura and urticaria. Laboratory tests would be particularly valuable in elucidating drug induced disease syndromes like erythema multiforme, erythema nodosum, glandular fever (from anti-tuberculous drugs) and systemic lupus erythematosus (from apresoline) -all presumably mediated by an allergic mechanism. In addition to the apresoline reaction, evidence of an immunological basis for lupus erythematosus is accumulating, especially since the finding of auto-antibodies to desoxyribosenucleic acid. But all the serological abnormalities in this disease could still be only secondary effects produced by some undiscovered causal agent.

\section{Granulomas}

Renewed interest in the allergic nature of the sarcoid granuloma has been aroused by the appearance of an unusual dermatosis. An eruption of small papules with the histology of a sarcoidal granuloma was found in the axillae of certain patients who had used a new type of stick deodorant containing a zirconium salt (Shelley and Hurley, 1958). More than 70 cases were described including one from England (Calnan and Sarkany, 1958) before the products were withdrawn by the manufacturers. The condition was produced experimentally and was shown to be due to specific hypersensitivity to the metal zirconium when injected intradermally in high dilution. The patients did not give a positive patch test. The only other metals known to produce sarcoid-like granulomas are mercury, silicon and beryllium. There is no evidence as yet that the silica granuloma, arising many years after silica had been accidentally introduced into the skin, is an allergic process. But berylliosis or beryllium disease, whether it is manifest as pulmonary infiltration or cutaneous sarcoidal nodules, is dependent on hypersensitivity to the metal. It can be demonstrated by a positive patch test reaction with a I per cent. solution of a beryllium salt, and Sneddon (1958) has shown that the eczematous response may merge after some weeks into a dermal granuloma. Patients with idiopathic sarcoidosis, pulmonary anthraco-silicosis as well as control subjects do not show hypersensitivity to intradermal injections of the salts of any of the elements in the periodic table (Shelley et al., 1958). 
The differential diagnosis of a sarcoidal granuloma in the skin may often be difficult because of the wide range of possible causes (Shelley and Hurley, 1958). The principal ones giving rise to this problem in England are tuberculosis, syphilis, leprosy, leishmaniasis, as well as silica, mercury, and other foreign bodies; most patients with a sarcoid reaction, however, fall into the category of idiopathic sarcoid, whose status as an independent entity is not universally accepted although the Kveim reaction specificity is strong evidence in its favour.

\section{Physical and Vascular Allergy}

There are a wide variety of dermatoses which are usually regarded as being of allergic origin, although this cannot yet be regarded as proven. One example is hypersensitivity to physical agents, such as light, heat and cold, some of which may be drug induced. In some instances of solar urticaria only have antibodies been demonstrated by the passive transfer technique. Substances such as rose bengal, eosin, and haematoporphyrin can greatly increase the reaction of the skin to sunlight without any sign of an allergic mechanism. A distinction has been made between phototoxic and photoallergic reactions, although it is not always easy to differentiate them, especially in the case of some drug photosensitivities like sulphanilamide (Blum, I94I) and chlorpromazine (Calnan, 1958). Although much work has been done to determine the active wavelengths of light in photosensitivity, with the aid of filters or a monochromator, we are still quite unable to define the nature of the allergen which is presumably formed in the skin by the incidcent light, if the reaction is to be regarded as a genuinely allergic one.

A number of skin disorders in which the main histological feature is inflammation and damage to small blood vessels, which cannot be explained in any other way, are now thought to be allergic, and the concept of vascular allergy or allergic vasculitis has arisen. Examples are Nodular Dermal Allergide of Gougerot (I95I), arteriolitis allergica (Ruiter, I953), cutaneous periarteritis rodosa (Miescher, 1956) and the median nasal granuloma associated with renal lesions. The chief evidence in favour of an allergic basis for these conditions is the similarity of the histological findings to known allergic lesions. Evidence of a causal allergen such as acute or focal bacterial in- fection, or drugs is unconvincing, and the patients are not usually responsive to treatment. These conditions are clinically recognizable, but their allergic basis remains questionable.

\section{REFERENCES}

ACKROYD, J. F. (1949), Clin. Sci., 7, 249.

ADAMSON, H. G., and MACLEOD, J. M. H. (1926), Brit. $\mathcal{F}$. Derm., 38, 192.

BLUM, H. F. (I94I), 'Photodynamic Action and Diseases caused by Light,' Reinhold, New York.

CALNAN, C. D., and SARKANY, I. (1957), Trans. St. Fohn's Hosp. Derm. Soc., 39, 28.

CALNAN, C. D., and SARKANY, I. (1958), Brit. F. Derm., 70, 435. CHASE, M. W. (1954), Int. Arch. Allergy, 5, 163.

COCA, A. F., WALZER, M., and THOOMEN, A. A. (I93I), 'Asthma and Hay Fever in Theory and Practice,' Charles C. Thomas, Springfield, III.

COOKE, R. A. (1944), F. Allergy 15, 203

ENGMAN, M. F., WEISS, R. S., and ENGMAN, M. F. Jr. (1936), Med. Clin. N. Amer., 20, 651.

EPSTEIN, S. (1956), Dermatologica (Basel), Ir3, 191.

EPSTEIN, S. (1958), Ibid., II7, 287.

EPSTEIN S. and KLIGMAN, A. (1957), F. invest. Derm. 28, 291

FREY, J. R., and WENK, P. (1956), Ibid., 112, 265.

GANS, O. (1956), Trans. St. Fohn's Hosp. Derm. Soc., 37, r.

GOUGEROT, H. (I95I), Minerva Dermatologica, XXVI I.

HAMILTON, L. D. (1958), N.Y. Acad. Sci., 73 (1), 39.

HARRIS, T. N., HARRIS, S., and FARBER, M. B. (1956), F. exp. Med., 104, 663 .

HAXTHAUSEN, H. (1939), Acta derm =venereol (Stockh.), 20, 257. HERRMANN, F. (1946), Ann. Allergy, 4, 4.

HOIGNE, R., GROSSMANN, W., and STORCK, H. (1956) Int. Arch. Allergy, 8, 103.

INDERBITZEN, Th. (1955), Int. Arch. Allergy, 7, 140.

JADASSOHN, W. (1957), Int. Arch. Allergy, 11, 20.

KIERLAND, R. R. (1955), Amer. Practit. (Philad.), 6, 1089.

KLIGMAN, A. M. (1958), Arch. Derm., 77, 149.

KLIGMAN, A. M. (1958), Ibid., 78, 47, 359.

LANDSTEINER, K., and CHASE, M. W. (1942), Proc. Soc. exp. Biol., 49, 688.

MAYER, R. L. (1957), Int. Arch. Allergy, r1, r.

MIESCHER, G. (1956), Derm. Wschr., 134, 1275.

O'LEARY, P. A. (1953), Sth. med. F. (Bgham., Ala.), 46, 67.

PAPPENHEIMER, A. M. (1958), The Harvey Lectures, Academic Press, New York.

PATON, W. D. M. (I956), in 'Histamine' CIBA Foundation Symposium, Little, Brown \& Co., Boston, Mass.

PEPYS, J. (1951), Int. Arch. Allergy, 2, 147.

PEPYS, J. (1953), Postgrad. med. F., 29, 351, 564.

RILEY, J. F., and WEST, G. B. (1953), F. Physiol. (Lond.), 120, 528 ROSTENBERG, A., Jr. (1955), Amer. Practit. (Philad.), 6, 1094. RUITER, M. (1953), Brit. f. Derm., 65, 77.

SCHNYDER, U. W. (1957), Int. Arch. Allergy, r1, 64.

SHELLEY, W. B., and ARTHUR, R. P. (1955), Arch. Derm. and Syph., 72, 399.

HURLEY, H. J. (1958), Brit. F. Derm. SHELLEY, W. B., et al. (1958), F. invest. Derm., 31, 301.

SNEDDON, I. B. (1958), Postgrad. med. F., 34, 262.

STRAUSS, J. S., and KLIGMAN, A. M. (I957), Arch. Derm.

SULZBBERGER, M. B. (1940), 'Dermatologic Allergy,' Charles C. Thomas, Springfield, Ill.

TUFT, L. (1940), Ұ. invest. Derm., 12, 211.

VON PIRQUET, C., and SCHICK, B. (1905), 'Die Serumkrankheit,' Deuticke, 'Vienna.

WILSON, H. T. H. (1958), Brit. med. F., i, 1378.

WINKELMANN, R. K. (1957), Proc. Staff Meetings of the Mayo Clinic, 32, 329.

ZIMMERMAN, M. C. (1959), Arch. Derm., 79, I.

Bibliography continued from pàge 444-Kenneth M. Citron, M.D., M.R.C.P.

CITRON, K. M., FRANKLAND, A. W., and SINCLAIR, J. D. (1958), Thraox, 13, 229.

GANDEVIA, B., and HUGH-JONES, P. (1957), Ibid., 12, 290.

GANDEVIA, B., HUME, K. M., and PRIME, F. J. (1957), Lancet, i, 956 .
HERSCHFUS, J. A., BRESNICK, E., and SEGAL, M. S. (1953), Amer. F. Med., 14, 23, 34 .

SEGAL, M. S., and ATTINGER, E. O. (r957), ' Clinical Cardiopulmonary Physiology,' Grune and Stratton, New York.

THOMPSON, W. B., and HUGH-JONES, P. (1958), Brit. med. $₹$., i, 1093 . 